\title{
Impact of Neurodevelopmental Delays While Treating Substance Abuse
}

\author{
Ami Norris Brilliant ${ }^{1 *}$ and Joshua King ${ }^{2}$ \\ ${ }^{1}$ Department of psychology, USA \\ ${ }^{2}$ Department of psychology Center for Motivation and Change, USA
}

*Corresponding author: Ami Norris Brilliant, Department of psychology, USA.

Received Date: July 31, 2019

Published Date: August 29, 2019

\section{Opinion}

There is ample research about the stigma that is associated with substance use disorders [1]. It is far reaching and impacts many aspects of a person's experience not only with their interest in seeking treatment, but also in the quality of treatment they are provided. Because of the stigmatization of the substance user, many, if not all, of their behaviors are attributed to the person, and underlying comorbid issues are not considered as a potential cause of factor that is maintaining substance use behaviors [2].

What we do know is that people with substance use disorders are rarely struggling with just substances in the absence of other disorders. There is research that up to $50 \%$ [JK- check this statistic] of all people with a substance use disorders also qualify for post traumatic stress disorder. This is also true for other mental health problems such as bipolar disorder and schizophrenia [3]. And yet, even with this information, the separation of healthcare and addiction treatment services means that we are not treating these symptoms together [4]. This means that despite knowledge that substance use disorders and other co-occurring mental health issues are highly intertwined, they are not being treated together, and are often being overlooked in one setting or the other.

One area where this happens regularly is the overlap of substance use disorders with neurodevelopmental delays, such as ADHD and autism, even though the evidence that they are highly connected is robust. In one study, $23.1 \%$ of clients who qualified for a substance use disorder also met criteria for comorbid ADHD diagnosis [5]. In another 30\% of treatment seeking adults with autism had comorbid substance abuse. There is little research on substance abuse and traditional learning disorders, which only highlights the need for more integrated understanding of the impact that learning differences have on substance use.
Clinicians can be too quick to assume that a client's forgetfulness or their lack of organization or their lack of motivation is due to their substance use, and ignore the significant data that shows the role that these neurodevelopmental delays have on the decision making process. Obviously not all treatment seeking individuals need a full blown neuropsychological evaluation. However, we believe that it is very important for clinicians to look at neuropsychological sequalae of neurodevelopmental delays as they begin their initial assessment of their clients.

They should also keep this in mind as they continue treatment. When a client continues to miss deadlines, demonstrate poor planning, and impulsive behaviors (like impulsive substance use) continue to crop up, clinicians should look into neuropsychological assessment for their clients much more regularly than they do. This will not only provide clients with a higher level of treatment, but it will help clinicians have a better understanding of how their clients are making behavioral choices. This may even reduce therapist burnout, as client behavior may make more sense and the clinician can build more empathy for their client.

Finally, there are clear cognitive deficits that come with long term substance abuse and if the clinician is aware of the individual profiles of each of their patients, it may affect their treatment planning positively. Neuropsychological assessment can also help the clinician ascertain if the treatment seeking individual is utilizing their substance abuse to self medicate an issue that can be remediated by skills or appropriate medication management. It is our opinion that neuropsychological evaluation should be used much more frequently with this particular population.

\section{Acknowledgment}

None. 


\section{Conflict of Interest}

Author declare no conflict of interest.

\section{References}

1. Avery JD, Avery JJ (2019) The stigma of addiction: An essential guide. Springer.

2. Peele S (1987) A Moral Vision of Addiction: How People's Values Determine Whether They Become and Remain Addicts. Journal of Drug Issues, 17(2): 187-215.
3. Hartz SM, Pato CN, Medeiros H, Cavazos Rehg, P, Sobell JL, et al. (2014) Comorbidity of Severe Psychotic Disorders with Measures of Substance Use. JAMA Psychiatry 71(3): 248-254.

4. Padwa HG, Guerrero ET, Braslow JM, Fenwick KU (2015) Barriers to Serving Clients with Co-occurring Disorders in a Transformed Mental Health System. Psychiatr Serv 66(5): 547-550.

5. van de Glind G (2013) ADHD in treatment seeking patients with a substance use disorder. 's-Hertogenbosch: Boxpress, pp. 231. 